\title{
ARTÍCULO DE REVISIÓN \\ Aproximación a los metanálisis de comparación de múltiples tratamientos: una revisión de la literatura
}

Fecha de recibido: 16 de junio de 2018 .

Fecha de aprobación: 26 de noviembre de 2018.
Forma de citar este artículo: Zuluaga M, Vanegas D, Donado JH. Aproximación a los metanálisis de comparación de múltiples tratamientos: una revisión de la literatura. Med UPB. 2019;38(2):158-167. DOI:10.18566/medupb.v38n2.a08

1 Universidad CES. Medellín, Colombia.

2 Universidad Pontificia Bolivariana. Medellín, Colombia.

3 Hospital Pablo Tobón Uribe. Medellín, Colombia.

Dirección de correspondencia: Mateo Zuluaga Gómez. Correo electrónico: mateozg92@ hotmail.com
Approach to multiple treatment comparison meta-analyses: A review of the literature / Aproximação aos metanálise de comparação de múltiplos tratamentos: uma revisão da literatura

Mateo Zuluaga Gómez ${ }^{1}$ Daniel Vanegas Isaza², Jorge Hernando Donado Gómez ${ }^{2,3}$

\section{RESUMEN}

Los metanálisis de comparación de múltiples tratamientos permiten estudiar, a partir de revisiones sistemáticas, las comparaciones realizadas de forma directa o indirecta, sobre diversos tipos de intervenciones, con datos de varios ensayos clínicos que, por su homogeneidad en los resultados, pueden agruparse en un resultado común y expresable de manera cuantitativa. Otros nombres que recibe son: metanálisis en red, metanálisis de comparación mixta o metanálisis indirectos. Al permitir la comparación de varios tratamientos facilitan una mejor estimación del efecto de las intervenciones, lo que guía de forma más certera al clínico en sus decisiones y en la aplicación de la información en el cuidado de sus pacientes. El objetivo de esta revisión es guiar al lector para que realice de manera organizada y adecuada la lectura crítica (según recomendaciones JAMA) de los metanálisis de comparación de múltiples tratamientos. Este artículo está orientando a los autores y a los editores, y contiene información sobre cómo deben interpretarse y comunicarse estos estudios.

Palabras clave: metanálisis como asunto; terapéutica; práctica clínica basada en la evidencia

\begin{abstract}
Multiple treatment comparison meta-analyses are systematic reviews that allow to make direct or indirect comparisons of different interventions from the data collection results of randomized clinical trials, grouping them in a common outcome or conglomerate which is expressed quantitatively. Other names given are network meta-analyses, or mixed treatment comparison meta-analyses or indirect meta-analyses. The use of the comparison of several treatments allows a greater estimation of the intervention effect. This is a guide for the physician to make more accurate decisions and to apply better care criteria to their patients. This literature review should help the reader to perform an organized and adequate critical reading (according to the JAMA recommendations) of the multiple treatment comparison meta-analyses. The article is addressed to authors and editors, and it contains information on how this type of studies should be interpreted and communicated.
\end{abstract}

Key words: meta-analysis as topic; therapeutics; evidence-based practice

\section{RESUMO}

Os metanálise de comparação de múltiplos tratamentos permitem estudar, a partir de revisões sistemáticas, as comparações realizadas de forma direta ou indireta, sobre diversos tipos de intervenções, com dados de vários ensaios clínicos que, por sua homogeneidade nos resultados, podem agrupar-se num resultado comum e de expressão 
de maneira quantitativa. Outros nomes que recebe são: metanálise em rede, metanálise de comparação mista ou metanálise indiretos. Ao permitir a comparação de vários tratamentos facilitam uma melhor estimação do efeito das intervenções, o que guia de forma mais certeira ao clínico em suas decisões e na aplicação da informação no cuidado de seus pacientes. O objetivo desta revisão é guiar ao leitor para que realize de maneira organizada e adequada a leitura crítica (segundo recomendações JAMA) dos metanálise de comparação de múltiplos tratamentos. Este artigo está orientando aos autores e aos editores, e contém informação sobre como devem interpretar-se e comunicar-se estes estudos.

Palavras chave: metanálise como assunto; terapêutica; prática clínica baseada na evidência

\section{INTRODUCCIÓN}

Los ensayos clínicos aleatorizados (RTC por su sigla en inglés) permiten analizar el efecto de una intervención en un grupo de pacientes con una misma enfermedad. A los pacientes se les asigna de forma aleatoria a un grupo de tratamiento o a uno de control (o placebo), para conocer si la intervención es mejor o igual, pero no inferior al tratamiento con el que se está comparando ${ }^{1}$. Por ejemplo, los ensayos permiten asegurar que al añadir colchicina a la terapia antiinflamatoria convencional para la pericarditis aguda, reduce la incidencia de pericarditis recurrente o incesante $(37,5 \%$ vs $16,7 \%$, reducción del riesgo relativo $-\mathrm{RRR}-\mathrm{del}$ 0,56; intervalo de confianza del $95 \%$ -IC95\%- =0,3-0,72; número de pacientes necesario a tratar $-\mathrm{NNT}-=4 ; \mathrm{p}<0,001)^{2}$, o que la aspiración del trombo de forma rutinaria, antes de la intervención coronaria percutánea (ICP) en pacientes con elevación del segmento ST (STEMI), no reduce la tasa de muerte por cualquier causa ni la rehospitalización por infarto o la trombosis del stent al año, cuando se compara con ICP individualizada ${ }^{3}$.

Cuando se realiza una revisión sobre un tema específico, se puede encontrar que se han desarrollado diversos tipos de estudios (ensayos clínicos, cohortes, casos y control, transversales, entre otros), que evalúan una misma intervención/exposición, con desenlaces similares, en una población con iguales características y con resultados dispares entre los trabajos. Las revisiones sistemáticas sintetizan de forma cualitativa la información de todos estos reportes en uno solo. Los metanálisis que generalmente se realizan después de una revisión sistemática, permiten el reporte cuantitativo de la información. Ambos tipos de estudios se consideran de mayor evidencia clínica, para un tratamiento o intervención preventiva ${ }^{1}$.

Los metanálisis de comparación de múltiples tratamientos (MTC por su sigla en inglés) permiten comparar de forma indirecta algún tipo de intervención, sin que sea necesario realizar un ensayo nuevo. Supongamos que un investigador requiere ponderar el medicamento A contra el B y que, en dicho caso, no es posible realizar un ensayo clínico que valore directamente los fármacos (por razones de costos o de logística), pero que en la revisión de la literatura se dan cuenta de que existen estudios sobre el medicamento A contra placebo y otros sobre el medicamento $\mathrm{B}$ contra placebo. Un MTC permite evaluar de forma directa o indirecta distintas intervenciones para una misma patología, realizadas en diferentes ensayos, donde ninguno de los artículos primarios (los RCT) las abordan de forma simultanea ${ }^{1,4}$. En el MTC hay cabida para las comparaciones directas (A vs. B) y para las indirectas ( $\mathrm{A}$ vs. $\mathrm{C}$ y $\mathrm{B}$ vs. $\mathrm{C}$ ), que refuerzan las inferencias realizadas a partir de los resultados de los estudios primarios ${ }^{4,5}$.

Otros nombres que pueden adoptar los MTC son metanálisis en red (pues involucran una red de diferentes tratamientos), metanálisis de comparación mixtos o metanálisis indirectos. Estos nombres dan cuenta de que los mencionados trabajos tienen en consideración comparaciones que ocurren en el mismo momento o sobre 
tratamientos múltiples, que se pueden realizar de forma directa o indirecta ${ }^{1,4,6}$.

Este tema es nuevo y en los últimos años se ha disparado el número de MTC. De hecho, no existe aún un término para estos trabajos en el Index Medicus. En la búsqueda en PubMed, mediante los términos en el Título/Abstract: indirect meta-analysys, multiple treatment comparisons meta-analysis, multiple treatment comparisons meta-anlyses, network meta-analysis, para artículos publicados entre el primero enero del año 2000 y el 30 de septiembre de 2018, se encontró un comportamiento exponencial desde el año 2007 (Figura 1).

A continuación, se realiza una revisión acerca de los aspectos del diseño metodológico de los MTC y sobre cómo realizar una lectura crítica según las recomendaciones JAMA.

\section{Aspectos del diseño metodológico de los MTC}

¿Cómo se debe evaluar el diseño del estudio?

Al plantearse un MTC, el investigador debe tener presente los siguientes tres interrogantes en cuanto el diseño ${ }^{1}$ :

- ¿Son los estudios homogéneos para combinar dicha intervención?

- ¿Son similares, con excepción en la intervención, que les permita ser comparados?

- ¿Son los resultados entre las intervenciones comparadas (directa o indirectamente) lo suficientemente confiables?

\section{Homogeneidad}

La homogeneidad es la falta de variabilidad de los estudios que hacen parte del MTC, con respecto al resultado promedio global. Este cálculo se puede evaluar

Figura 1. Comportamiento anual de las publicaciones de metanálisis de comparación de múltiples tratamientos, entre el año 2000 y el año 2018.

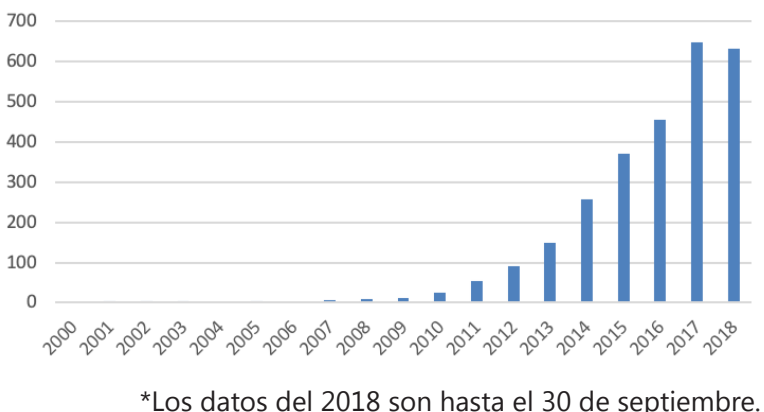

a través del índice de inconsistencia $\left(\mathrm{I}^{2}\right)$, la prueba $\mathrm{Q}$ de Cochrane o el tau ${ }^{2}$. La importancia de dicho valor es que entre mayor heterogeneidad, será menor la credibilidad del resultado del metanálisis ${ }^{1,5}$.

Al realizar un metanálisis, ya sea de comparaciones directas o indirectas, es importante asumir que haya similitud entre las características clínicas y metodológicas de los ensayos clínicos. Es fundamental que el tipo de pacientes, la intervención, la duración del seguimiento, las estrategias de medición y las covariables hayan sido considerados de modo similar por los investigadores en cada estudio primario ${ }^{7}$.

\section{Consistencia o coherencia}

La consistencia o coherencia se refiere a la dirección que tienen los resultados de comparaciones directas y directas, ello quiere decir que no pueden ser divergentes. Cuando existen inconsistencias (incoherencias), se debe tener en cuenta si hubo errores en la validez interna de los estudios seleccionados o heterogeneidad elevada, en cuanto a la variabilidad de los hallazgos.

\section{Estimación global}

Para la estimación global de efecto por medio de un metanálisis se utilizan dos modelos: efectos fijos y efectos aleatorios ${ }^{7}$. En el primer caso se asume que la magnitud o tamaño del efecto es el mismo en todos los estudios y que la variabilidad se produce por azar ${ }^{5,8}$. En el modelo de efectos aleatorios hay diversidad en los resultados de los distintos ensayos, por diferencias entre ellos y que son atribuidas a las características de los pacientes, entre otras. Los hallazgos obtenidos con los diferentes modelos coinciden en caso de homogeneidad de los estudios.

Cuando los estudios no tienen una metodología o unas características basales que permitan su comparación, no es posible sintetizar de forma cuantitativa los resultados y así el estudio pasaría a ser una revisión sistemática ${ }^{9}$.

Este tipo de estudio estima su validez en supuestos, es decir, la legitimidad de lo que se va a comparar de forma indirecta depende de la calidad de los estudios que se hayan incluido. La evidencia indirecta es de carácter observacional, ello quiere decir que el investigador basado en su conocimiento tiene en cuenta las intervenciones a evaluar y, así mismo, los diseños de estudio a utilizar?

\section{Coherencia}

La coherencia en los MTC se refiere al nivel de acuerdo que hay entre comparaciones directas o indirectas. Es recomendable que el acuerdo sea grande para que permita la obtención de un bien estimativo combinado. En este respecto, son importantes los criterios de selección de 
pacientes y el control de cointervenciones realizados en los ensayos seleccionados ${ }^{7,9}$.

\section{Geometría en red}

Hay distintas formas de diseñar las comparaciones en los MTC, es decir, que existen diferentes patrones de ponderación de los tratamientos, conocidos como geometría en red. Entre los tipos de geometría están los siguientes (Figura 2) ${ }^{1,8}$ :

- Red en estrella / Red de asa abierta: todas las intervenciones tienen un comparador en común. Ejemplo: el fármaco A se puede comparar con el fármaco B, C, D, E, F y G. El comparador común más frecuente es el placebo.

- Red en asa cerrada: en este tipo se comparan tres intervenciones distintas y, a través del mismo, se pueden obtener datos para hacer comparaciones de forma directa o indirecta.

- Red conectada: las intervenciones que se comparan en este MTC, se hacen unas con otras en varios ensayos clínicos.

- Red compleja: este tipo de diseño permite que haya múltiples asas y brazos, permitiendo conexiones de forma dispersa al momento de comparar las intervenciones.

La representación gráfica de una red permite mostrar los distintos tipos de tratamiento evaluados, para ayudar a comprender y determinar cuál es la fuerza de la evidencia para cada comparación. Además, este modo de presentación realza la transparencia de los MTC. Para representar cada tratamiento se usan nodos y para señalar o relacionar estudios que se comparan, se utilizan líneas o conexiones entre ellos. Es importante mencionar que entre los software estadísticos más utilizados para MRC están Stata y WinBUGS, con distintos comandos de análisis y formas de realizar la representación gráfica de los estudios ${ }^{9-13}$.

En cada red es necesario evaluar la asimetría, es decir, el peso con el que una comparación específica de algún tipo de intervención se representa en la red.

\section{Análisis de los resultados}

Los resultados de los MTC se pueden interpretar según las recomendaciones del grupo $\mathrm{JAMA}^{1}$, que ayudan a analizar la validez interna, los resultados en sí y la aplicabilidad de los mismos en la población. Recientemente, el grupo Grading of Recommendations Assessment, Development and Evaluation (GRADE por su sigla en inglés) ${ }^{14}$ desarrolló una serie de aproximaciones y recomendaciones sobre la calidad de la evidencia, de acuerdo a ciertos ítems que garantizan que el lector puede confiar en el efecto demostrado sobre una comparación específica. GRADE plantea que, si todos los ensayos clínicos tienen bajo riesgo de sesgo, el metanálisis se puede aplicar en la práctica siempre y cuando se haya incluido la población adecuada para la intervención administrada y que la precisión del efecto sea correcta, sin riesgos importantes de sesgos de publicación.

Específicamente, el grupo GRADE sugiere que en los MTC se presenten los efectos directos e indirectos con los intervalos de confianza del $95 \%$ y que se tenga en cuenta el estimado directo (que se obtiene a partir de la valoración frente a frente o medicamento a medicamento, es decir, medicamento A vs. medicamento B) y el indirecto que se obtiene de varias comparaciones, contra común una misma intervención. Después de esto, se debe puntuar la calidad del efecto directo e indirecto (se tiene en cuenta si es de alta, moderada, baja o muy baja). Luego se debe presentar el estimado del MTC para cada comparación y puntuar su calidad.

Figura 2. Ejemplos de geometría en red.

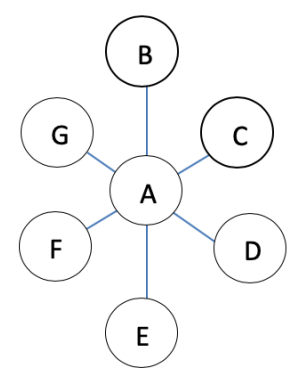

Red en estrella

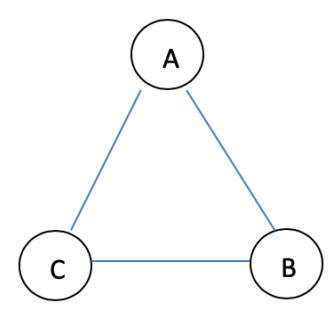

Red en asa abierta

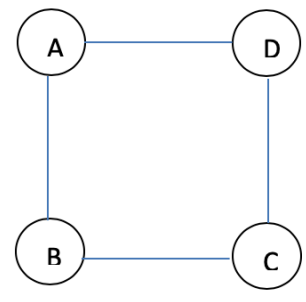

Red conectada 


\section{¿La variabilidad puede afectar los resultados?}

En algunos casos la variabilidad puede afectar los resultados, pero en dicha situación, el investigador debe indagar por las siguientes cuestiones en los estudios seleccionados:

- ¿Los ensayos clínicos incluidos tenían una magnitud similar del efecto?

- ¿El tiempo de seguimiento de los pacientes fue similar en los estudios?

- ¿Se consideró el uso de terapia adyuvante?

- ¿Las dosis de la terapia a evaluar fueron similares?

\section{Ejemplos de MTC}

Existen publicaciones de MTC en áreas como reumatología ${ }^{15-21}$, psiquiatría ${ }^{22-27}$, oncología ${ }^{28-30}$, oftalmo$\log i^{31-33}$, cardiología ${ }^{34-38}$, cirugía $^{33}$, dermatología ${ }^{39-42}$, neumología $\mathrm{a}^{43-47}$, medicina interna ${ }^{48-52}$, urología ${ }^{53}$, ortopedia $^{54-55}$, ginecología ${ }^{56-57}$ o nefrología ${ }^{58-59}$.

Uno de los estudios realizados en oncología compara la seguridad y eficacia entre los regímenes de quimioterapia para tratamiento avanzado de cáncer pancreático. Con este fin se realizó una búsqueda en MEDLINE, PubMed, EMBASE y Cochrane (período 2002-2013), teniendo en cuenta investigaciones que evaluaran desenlaces como supervivencia total, neutropenia febril grado 3-4, síntomas gastrointestinales y neuropatía sensitiva. En total, se incluyeron 23 reportes, con 19 tipos de regímenes quimioterapéuticos en 9989 pacientes. Se realizó un metanálisis en red (estrella), al no haber comparaciones directas y se encontró que algunas combinaciones de terapias pueden ser más beneficiosas en el tratamiento de cáncer pancreático avanzado ${ }^{28}$.

Otro de los ejemplos está en el área de cardiología. Se trata de un metanálisis con modelo bayesiano, que evalúa si la revascularización mejora el pronóstico más que el tratamiento médico en pacientes con enfermedad coronaria estable. Este estudio incluye ensayos reportados en MEDLINE y EMBASE, entre 1980 y 2013, que compararan la revascularización con el tratamiento médico. En él se encontró que el puente de la arteria coronaria disminuye el riesgo de muerte, el infarto del miocardio y las subsecuentes revascularizaciones, en mayor medida que el tratamiento médico. El análisis del MTC compara cada estudio primario entre sí, por lo que se realizó una red compleja, donde se permiten conexiones dispersas entre los grupos ${ }^{34}$.

\section{Ventajas de la aplicación de los MTC}

Los MTC resumen la evidencia sobre los efectos de una intervención, mediante la ponderación en red de los resultados de los ensayos. Entre otras ventajas: permiten realizar comparaciones directas e indirectas, brindando mayor precisión en la estimación de efectos de algún tratamiento ${ }^{1}$ y aumentan el poder estadístico, cuando combinan la evidencia directa e indirecta ${ }^{5,60}$. Dependiendo de los datos disponibles, las comparaciones directas o indirectas pueden mostrar distintos resultados, por ejemplo, cuando se hacen cotejos directos con un comparador en común (modelo en estrella). Si la complejidad de la geometría del MTC es mayor, hay más evidencia y se reduce la variabilidad en los resultados ${ }^{5}$.

Entre las técnicas de las comparaciones que se pueden realizar en los MTC se incluye el método de análisis bayesiano que, a partir de la interpretación de probabilidades, mide la magnitud de alguna de las intervenciones y diferencia si es superior que otra ${ }^{9}$.

Los MTC permiten clasificar las intervenciones de acuerdo con la probabilidad que tienen de ser el mejor tratamiento para un paciente en cierta condición. En este punto es importante tener en cuenta el tamaño del efecto mostrado por una intervención concreta ${ }^{8}$.

El uso, la interpretación y la aplicación apropiados de los MTC permiten a la comunidad médica guiar decisiones clínicas necesarias para la atención de sus pacientes ${ }^{1,5,6,8}$.

\section{Limitaciones o desventajas de los MTC}

Por tratarse de una técnica reciente y poco conocida por el gremio médico, la interpretación de la metodología y de los resultados de los MTC puede parecer difícil, pero se ha visto que con el pasar de los años, estos metanálisis han ganado aceptación y su número viene en aumento ${ }^{5,61}$.

Los MTC de ensayos clínicos que incluyeron muestras pequeñas son más susceptibles a sesgos y muestran falsos efectos, lo que puede sobreestimar o subestimar el impacto de un tratamiento con respecto a otro (sobre todo si se trata de una comparación directa con poca precisión). De ello se deduce que la muestra tiene un valor importante, puesto que influye en la precisión de los resultados ${ }^{5,62}$.

Cuando los MTC incluyen ensayos de diseño cruzado (cross-over, en los que un paciente cambia de brazo de intervención) se debe tener en cuenta, al momento de realizar el análisis, que los beneficios de la aleatorización se pueden perder en quienes cambiaron de tratamiento. Si el número de pacientes es alto, el análisis de supervivencia global puede estar sesgado y, por lo tanto, tiene el potencial de invalidar los resultados. Al incluir este tipo de ensayos en los MTC, se crea un dilema subjetivo en cuanto a la decisión que se llegue a tomar respecto a un resultado y si es aplicable o no ${ }^{5}$.

En los MTC se debe tener en cuenta la consistencia y la transitividad (es decir, si el medicamento $\mathrm{B}$ es mejor que $\mathrm{A}$ y $\mathrm{A}$ es mejor que $\mathrm{C}$, entonces, $\mathrm{B}$ es mejor que $\mathrm{C}$ ), tener esto claro evita las malas interpretaciones sobre este tipo de trabajos ${ }^{63}$. 


\section{Lectura crítica de los MTC}

En la presente revisión se siguen las recomendaciones de la guía de Mills et al., publicada en JAMA (debido a que esas recomendaciones reconocen la importancia del diseño epidemiológico y del análisis). Allí se proponen unos pasos para el análisis crítico. Las recomendaciones JAMA tiene en cuenta tres preguntas principales ${ }^{1}$ :

- ¿Son válidos los resultados del estudio?

- ¿Cuáles son los resultados?

- ¿Cómo puedo aplicar los resultados a mis pacientes?

A continuación, se dan las claves para responder a estas preguntas.

\section{¿Son válidos los resultados de estudio?}

\section{- ¿El estudio responde una pregunta específica?}

La pregunta de investigación tiene en cuenta estos componentes: pacientes, intervención, comparación y un desenlace o outcome (PICO). Los pacientes de cada ensayo clínico deben ser similares entre ellos y con los de los demás ensayos, en cuanto a: criterios de selección (inclusión y exclusión), intervención con dosis y vías de administración, cegamiento de la intervención y desenlaces evaluados. Se hace necesario explicar la variabilidad cuando los estudios son muy heterogéneos, mediante estrategias, como subgrupos o metaregresiones.

- ¿Se realizó una búsqueda exhaustiva de los estudios relevantes?

Lo ideal sería incluir todos los ensayos realizados hasta el momento. En este punto, los autores deberán explicar detalladamente en el protocolo, la forma en la que se realizó la selección de estudios primarios, tanto en las bases de datos como en otras fuentes.

- ¿Presentaban sesgos mayores los estudios primarios?

Se debe evaluar la validez interna o riesgo de sesgos de los ensayos. Es necesario analizar cómo se realizó la asignación aleatoria de los participantes, si hubo algunas pérdidas del seguimiento, si se realizaron análisis por intención de tratamiento y si estuvo oculta la intervención a evaluar. Es importante mencionar que los principales sesgos que presentan los MTC son el de publicación o los acontecidos, al reportar solo algunos desenlaces seleccionados. Con lo anterior podemos evaluar cuál es la validez interna del MTC y decidir si los resultados serán o no sesgados.

\section{¿Cuáles son los resultados?}

- ¿Cuál fue la cantidad de evidencia de la red de tratamiento?

La mejor manera de evaluar este ítem es tener en cuenta la cantidad de estudios primarios que fueron incluidos en la red de tratamiento y el número de eventos ocurridos en cada uno. También es importante evaluar cómo es la conexión entre ensayos, para lo que es necesaria la gráfica en red, mencionada en el punto sobre metodología. De esta manera el lector tendrá una mejor comprensión de las intervenciones comparadas y si fueron cotejadas de modo directo o indirecto (ver, Figura 1).

\section{- ¿Fueron similares los resultados entre los estudios?}

Cuando se encuentra una diferencia marcada entre las comparaciones directas de los resultados de los estudios, debe evidenciarse en los índices de homogeneidad $\left(\mathrm{I}^{2}\right)$. Se considera que son homogéneos los estudios con valores menores al $25 \%$, si los valores se encuentran entre el 25\% y el $50 \%$, se habla de heterogeneidad moderada. Aquellos con valores superiores al $50 \%$ tienen variabilidad alta. Según sea el grado de heterogeneidad será necesario realizar análisis por subgrupos o metaregresiones, e identificar las posibles razones por las que aparece la heterogeneidad. - ¿Fueron consistentes los resultados en la comparación directa e indirecta?

Se debe evaluar la consistencia o coherencia entre las comparaciones directas e indirectas (esto es válido para los diseños de asa cerrada o de red conectada, no para los que son en estrella). Si la prueba de incoherencia es negativa, hay fortaleza en las comparaciones realizadas. - ¿Cuáles fueron los efectos del tratamiento en general y su incertidumbre? ¿Cómo se ordenan los tratamientos?

Los resultados generales suelen presentarse con un estimado puntual del efecto, acompañado de un "intervalo de credibilidad" del 95\% (equivalente a un intervalo de confianza). Adicionalmente, se debe ordenar de menor a mayor el efecto que tenían las intervenciones. En lector debe ser cuidadoso con la interpretación de este punto, puesto que, en muchos casos, la diferencia entre dos intervenciones es mínima, como consecuencia de sesgos en el MTC.

- ¿Fueron los resultados robustos ante los supuestos de sensibilidad y sesgos potenciales?

Los autores deben realizar análisis de sensibilidad para observar cuánto pueden cambiar los resultados obtenidos al modificar algunos criterios preestablecidos.

\section{¿Cómo puedo aplicar los resultados a mi paciente?}

- ¿Fueron considerados todos los resultados importantes para el paciente?

Los MTC suelen reportar pocos desenlaces, incluso, en algunos casos, solo se evalúa un único desenlace. Los efectos adversos de las intervenciones no suelen estar incluidos en los MTC.

- ¿Fueron considerados todos los posibles tratamientos?

Es difícil incluir en un MTC todas las posibilidades terapéuticas disponibles para la patología en estudio. Es común dejar a un lado los estilos de vida (por ejemplo, 
dieta o ejercicio regular) y centrarse solo en los medicamentos, incluso, puede ser necesario solo incluir medicamentos de una familia o subfamilia (por ejemplo, betabloqueadores en el tratamiento de la falla cardíaca). - ¿Hay algún efecto en los subgrupos postulados que sea creíble?

Se deben tener precaución en la interpretación de los resultados en algún subgrupo de pacientes, debido a que pueden ser producto de un error tipo 1, por lo que es mejor analizarlos de forma exploratoria. Existen varios criterios para evaluar la credibilidad de un análisis de subgrupos. Entre estos criterios están que los análisis sean definidos a priori, en un número pequeño; que los subgrupos se identifiquen por características basales de los participantes y no mediante características medidas después de la asignación aleatoria; que la prueba de interacción sea estadísticamente significativa y que haya plausibilidad biológica ${ }^{64}$.

- ¿Cuál es, en general, la calidad de la evidencia y cuáles son sus limitantes?

Es importante evaluar la calidad y validez del MTC para determinar si los resultados son lo suficientemente fuertes para hacer inferencias. Lo ideal es que los MTC presenten pocos sesgos, que los resultados consistan en comparaciones directas e indirectas de estudios (donde no hubo control sin tratamiento), que los resultados de comparaciones directas e indirectas sean consistentes, que el efecto sea grande y que el intervalo de credibilidad sea estrecho.

\section{CONCLUSIONES}

Con esta revisión se buscó guiar al lector para la lectura crítica organizada y adecuada (de acuerdo con recomendaciones de JAMA) de los metanálisis de comparación de múltiples tratamientos. Se orienta a los autores y editores sobre cómo debe interpretarse y comunicar este tipo de estudios. Estos metanálisis son novedosos y permiten resumir la evidencia de distintos ensayos clínicos, que aún no se han comparado entre ellos. Existen distintas estrategias para guiar la lectura crítica, esta revisión realiza un abordaje según las recomendaciones de Mills et al.

\section{DECLARACIÓN DE CONFLICTO DE INTERESES}

Los autores declaran no tener ningún conflicto de intereses.

\section{REFERENCIAS}

1. Mills EJ, Ioannidis JP, Thorlund K, Schünemann HJ, Puhan M, Guyatt GH. How to use an article reporting a multiple treatment comparison meta-analysis. JAMA. 2012;308(12):1246-1253.

2. Imazio $M$, Brucato $A$, Cemin R, Ferua $S$. A Randomized trial of colchicine for acute pericarditis. N Engl J Med. 2013;369:1522-1528.

3. Lagerqvist B, Fröbert O, Olivecrona GK, Gudnason T, Maeng M, Alström P, et al. Outcomes 1 year after thrombus aspiration for myocardial infarction. N Engl J Med. 2014;371:1111-1120.

4. Lu G, Ades AE. Combination of direct and indirect evidence in mixed treatment comparisons. Stat Med. 2004;23:3105-3124.

5. Mills EJ, Bansback N, Ghement I, Thorlund K, Kelly S, Puhan M a, et al. Multiple treatment comparison meta-analyses: A step forward into complexity. Clin Epidemiol. 2011;3:193-202.

6. Cipriani A, Higgins JPT, Geddes JR, Salanti G. Conceptual and technical challenges in network meta-analysis. Ann Intern Med. 2013;159:130-137.

7. Glenny AM, Altman DG, Song F, Sakarovich C. Indirect comparisons of competing interventions. Health Technol Assess. 2005;9(26):1-134.

8. Mills EJ, Thorlund K, Ioannidis JPA. Demystifying trial networks and network meta-analysis. BMJ. 2013;2914:10-15.

9. Catalá F, Tobías A, Roqué M. Basic concepts for network meta-analysis. Aten Primaria. 2014;46(10):573-581.

10. White IR. Multivariate random-effects meta-regression: Updates to mvmeta. Stata J. 2011;11:255270 .

11. Jackson D, Riley R, White IR. Multivariate meta-analysis: potential and promise. Stat Med. 2011;30(20):2481-2498.

12. Catalá F, Tobias A, Cameron C, Moher D. Network meta-analysis for comparing treatment effects of multiple interventions: An introduction. Rheumatol Int. 2014;34(11):1489-1496.

13. Catalá $F$, Tobías A. Síntesis de la evidencia clínica y metaanálisis en red con comparaciones indirectas. Med Clin (Barc). 2013;140:182-187.

14. Puhan M, Schunemann HJ, Murad MH, Li T, Brignardello-Petersen R, Singh J, et al. A GRADE Working Group approach for rating the quality of treatment effect estimates from network meta-analysis. BMJ. 2014;349:g5630-g5630. 
15. Freemantle N, Cooper C, Diez-Perez A, Gitlin M. Results of indirect and mixed treatment comparison of fracture efficacy for osteoporosis treatments: A meta-analysis. Osteoporos Int. 2013;24(1):209-217.

16. Catalá F, SanFélix C, Tobías A, Hurtado I. Efficacy of osteoporosis therapies in a network meta-analysis with indirect comparisons: Many concerns for new tools of evidence synthesis? Osteoporos Int. 2013;24(6):1927-1928.

17. Jansen JP, Berqman GJ, Huels J, Olson M. The efficacy of bisphosphonates in the prevention of vertebral, hip, and nonvertebral-nonhip fractures in osteoporosis: A network meta-analysis. Semin Arthitis Rheum. 2011;40(4):257-284.

18. Tian SY, Feldman BM, Beyene J, Brown PE. Immunosuppressive therapies for the induction treatment of proliferative lupus nephritis: A systematic review and network metaanalysis. J Rheumatol. 2014;41(10):1998-2007.

19. Corbett MS, Rice SJ, Madurasinghe V, Slack R. Acupuncture and other physical treatments for the relief of pain due to osteoarthritis of the knee: Network meta-analysis. Osteoarthritis Cartilage. 2013;21(9):1290-1298.

20. McInnes IB, Nash P, Ritchlin C, Choy EH. Secukinumab for psoriatic arthritis: Comparative effectiveness versus licensed biologics/apremilast: A network meta-analysis. J Comp Eff Res. 2018;7(11):1107-1123.

21. Persson MSM, Stocks J, Walsh DA, Doherty M. The relative efficacy of topical non-steroidal antiinflammatory drugs and capsaicin in osteoarthritis: A network meta-analysis of randomised controlled trials. Osteoarthritis Cartilage. 2018;4584(18):31427.

22. Nussbaumer B, Morgan LC, Reichenpfader U, Greenblatt A, Hansen RA, Van Noord M, et al. Comparative efficacy and risk of harms of immediate-versus extended-release secondgeneration antidepressants: A systematic review with network meta-analysis. CNS Drugs. 2014;28(8):699-712.

23. Barth J, Munder T, Gerger H, Nuesch E. Comparative efficacy of seven psychotherapeutic interventions for patients with depression: A network meta-analysis. PloS Med. 2013;10(5):e1001454.

24. Riku Y, Atsuta N, Yoshida M, Tatsumi S. Differential motor neuron involvement in progressive muscular atrophy: A comparative study with amyotrophic lateral sclerosis. BMJ Open. 2014;4(5):e005213.

25. Rusinova K, Kukal J, Simek J, Cerny V. Limited family members/staff communication in intensive care units in the Czech and Slovak Republics considerably increases anxiety in patients' relatives-the DEPRESS study. BMC psychiatry. 2014;27(14):21.

26. Guo X, Duan X, Suckling J, Chen H, Liao W, Cui Q, et al. Partially impaired functional connectivity states between right anterior insula and default mode network in autism spectrum disorder. Hum Brain Mapp. 2019;40(4):1264-1275.

27. Cortese S, Adamo N, Del Giovane C, Mohr-Jensen C. Comparative efficacy and tolerability of medications for attention-deficit hyperactivity disorder in children, adolescents, and adults: A systematic review and network meta-analysis. Lancet Psychiatry. 2018;5(9):727-738.

28. Gresham GK, Wells GA, Gill S, Cameron C, et al. Chemotherapy regimens for advanced pancreatic cancer: A systematic review and network meta-analysis. BMC Cancer. 2014;14(1):471.

29. Leung HW, Chan AL, Lin SJ. Indirect comparisons of efficacy and safety between seven newer targeted agents for metastatic renal cell carcinoma: A network meta-analysis of randomised clinical trials. Mol Clin Oncol. 2014;2(5):858-864.

30. Yokoe T, Hayashida T, Nagayama A, Nakashoji A, Maeda H, Seki T, et al. Effectiveness of antiemetic regimens for highly emetogenic chemotherapy-induced nausea and vomiting: A systematic review and network meta-analysis. Oncologist. 2018;doi:10.1634/theoncologist.2018-0140.

31. Régnier S, Malcolm W, Allen F, Wright J. Efficacy of anti-VEGF and laser photocoagulation in the treatment of visual impairment due to diabetic macular edema: A systematic review and network meta-analysis. PloS One. 2014;9(7):e102309.

32. Ford JA, Shyangdan D, Ulthman OA, Lois N. Drug treatment of macular oedema secondary to central retinal vein occlusion: A network meta-analysis. BMJ Open. 2014;4(7):e005292.

33. Sangroongruangsri S, Ratanapakorn T, Wu O, Anothaisintawee T. Comparative efficacy of bevacizumab, ranibizumab, and aflibercept for treatmentof macular edema secondary to retinal vein occlusion: A systematic review and network meta-analysis. Expert Rev Clin Pharmacol. 2018;11(9):903-916.

34. Windecker S, Stortecky S, Stefanini G, daCosta B. Revascularisation versus medical treatment in patients with stable coronary artery disease: Network meta-analysis. BMJ. 2014;349:G4605.

35. Johnston BC, Kanters S, Bandayrel K, Wu P. Comparison of weight loss among named diet programs in overweight and obese adults: A meta-analysis. JAMA. 2014;312(9):923-933.

36. Naci H, Brugts JJ, Fleurence R, Ades AE. Dose-comparative effects of different statins on serum lipid levels: A network meta-analysis of 256,827 individuals in 181 randomized controlled trials. Eur J Prev Cardiol. 2013;20(4):658-670.

37. Mitchell SA, Simon TA, Jakouloff D, Orme ME. The efficacy and safety of oral anticoagulants in warfarin-suitable patients with nonvalvular atrial fibrillation: Systematic review and metaanalysis. Clin Appl Thromb Hemost. 2013;19(6):619-631. 
38. Palmerini T, Biondi-Zoccai G, Della Riva D, Mariani A, Sabaté M, Valgimigli M, et al. Clinical outcomes with drug-eluting and bare-metal stents in patients with ST-segment elevation myocardial infarction: Evidence from a comprehensive network meta-analysis. J Am Coll Cardiol. 2013;62(6):496-504.

39. Carroll C, Hummel S, Leaviss J, Ren S, Stevens JW, Cantrell A, et al. Systematic review, network meta-analysis and exploratory cost-effectiveness model of randomized trials of minimally invasive techniques versus surgery for varicose veins. Br J Surg. 2014;101(9):1040-52.

40. Vegter S, Tolley K. A network meta-analysis of the relative efficacy of treatments for actinic keratosis of the face or scalp in Europe. PloS One. 2014;9(6):e96829.

41. Rotta I, Ziegelmann PK, Otuki MF, Riveros BS. Efficacy of topical antifungals in the treatment of dermatophytosis: A mixed-treatment comparison meta-analysis involving 14 treatments. JAMA Dermatol. 2013;149(3):341-349.

42. Gupta AK, Charette A. The efficacy and safety of $5 \alpha$-reductase inhibitors in androgenetic alopecia: A network meta-analysis and benefit-risk assessment of finasteride and dutasteride. J Dermatolog Treat. 2014;25(2):156-161.

43. Cope S, Donohue JF, Jansen JP, Kraemer M, Capkun-Niggli G, Baldwin M, et al. Comparative efficacy of long-acting bronchodilators for COPD: A network meta-analysis. Respir Res. 2013;14:100

44. Loymans RJ, Gemperli A, Cohen J, Rubinstein SM. Comparative effectiveness of long term drug treatment strategies to prevent asthma exacerbations: Network meta-analysis. BMJ. 2014;348:G3009.

45. Cahill K, Stevens S, Perera R, Lancaster T. Pharmacological interventions for smoking cessation: An overview and network meta-analysis. Cochrane Database Syst Rev. 2013;5(CD009339).

46. Bakalos G, Miligkos M, Doxani C, Mpoulimari I, Rodopoulou P, Zintzaras E. Assessing the relative effectiveness and tolerability of treatments in small cell lung cancer: A network meta-analysis. Cancer Epidemiol. 2013;37(5):675-682.

47. Aziz MIA, Tan LE, Wu DB, Pearce F, Chua GSW, Lin L, et al. Comparative efficacy of inhaled medications (ICS/LABA, LAMA, LAMA/LABA and SAMA) for COPD: a systematic review and network meta-analysis. Int J Chron Obstruct Pulmon Dis. 2018;13:3203-3231.

48. Bansal M, Farrugia A, Balboni S, Martin G. Relative survival benefit and morbidity with fluids in severe sepsis - a network meta-analysis of alternative therapies. Curr Drug Saf. 2013;8(4):236-245.

49. Yeo YH, Hsu CC, Lee CC, Ho HJ, Lin JT, Wu MS, et al. Gastrointestinal disease and Helicobacter Consortium. Systematic review and network meta-analysis: Comparative effectiveness of therapies for second-line Helicobacter pylori eradication. J Gastroenterol Hepatol. 2018;34(1):5967.

50. Knottnerus BJ, Grigoryan L, Geerlings SE, Moll van Charante EP, Verheij TJ, Kessels AG, et al. Comparative effectiveness of antibiotics for uncomplicated urinary tract infections: Network meta-analysis of randomized trials. Fam Pract. 2012;29(6):659-670.

51. Bally M, Dendukuri N, Sinclair A, Ahern SP, Poisson M, Brophy J. A network meta-analysis of antibiotics for treatment of hospitalised patients with suspected or proven meticillin-resistant Staphylococcus aureus infection. Int J Antimicrob Agents. 2012;40(6):479-495.

52. Littlewood KJ, Higashi K, Jansen JP, Capkun-Niggli G, Balp MM, Doering G, et al. A network meta-analysis of the efficacy of inhaled antibiotics for chronic Pseudomonas infections in cystic fibrosis. J Cyst Fibros. 2012;11(5):419-426.

53. Zhu Z, Zhang J, Liu Y, Chen M, Guo P, Li K. Efficacy and toxicity of external-beam radiation therapy for localised prostate cancer: a network meta-analysis. Br J Cancer. 2014;110(10):2396-2404.

54. Krogh TP, Bartels EM, Ellingsen T, Stengaard-Pedersen K, Buchbinder R, Fredberg U, et al. Comparative effectiveness of injection therapies in lateral epicondylitis: A systematic review and network meta-analysis of randomized controlled trials. Am J Sports Med. 2013;41(6):1435.

55. Wang J, Wang J, Zhang K, Wang Y, Bao X. Bayesian network meta-analysis of the effectiveness of various interventions for nontraumatic osteonecrosis of the femoral head. Biomed Res Int. 2018;2018:2790163.

56. Daniels JP, Middleton LJ, Champaneria R, Khan KS, Cooper K, Mol BW, et al. Second generation endometrial ablation techniques for heavy menstrual bleeding: Network meta-analysis. BMJ. 2012;344:E2564.

57. Lv S, Yu J, Xu X. A comparison of effectiveness among frequent treatments of recurrent spontaneous abortion: A Bayesian network meta-analysis. Am J Reprod Immunol. 2018;80(1):e12856.

58. Farrokhi F, Abedi N, Beyene J, Kurdyak P, Jassal SV. Association between depression and mortality in patients receiving long-term dialysis: A systematic review and meta-analysis. Am J Kidney Dis. 2014;63(4):623-635.

59. Yang Y, Qiu S, Deng L, Tang X, Li X, Wei Q et al. Outcomes of bisphosphonate and its supplements for bone loss in kidney transplant recipients: A systematic review and network meta-analysis. BMC Nephrol. 2018;19(1):269

60. O'Regan C, Ghement I, Eyawo O, Guyatt GH, Mills EJ. Incorporating multiple interventions in meta-analysis: An evaluation of the mixed treatment comparison with the adjusted indirect comparison. Trials. 2009;10:86. 
61. Salanti G, Kavvoura FK, Ioannidis JP. Exploring the geometry of treatment networks. Ann Intern Med. 2008;148:544-553.

62. Thorlund K, Devereaux PJ, Wetterslev J, Guyatt G, Ioannidis JP, Thabane L, et al. Can trial sequential monitoring boundaries reduce spurious inferences from meta-analyses? Int J Epidemiol. 2009;38:276-286.

63. Catalá F, Hutton B, Moher D. La propiedad transitiva en los ensayos clínicos controlados: si B es mejor que $A$ y $C$ es mejor que $B$, ¿C será mejor que A? Rev Esp Cardiol. 2014;67(8):597-602.

64. Oxman A, Guyatt G. A consumer's Guide to subgroup analyses. Ann Intern Med. 1992;116(1):7884. 